\title{
COMUNICACIÓN PARA LA INCLUSIÓN SOCIAL DE MINORÍAS. LA CLASE MÁGICA, UN MODELO DE INTERVENCIÓN PARA EL CAMBIO SOCIAL
}

\author{
COMMUNICATION FOR SOCIAL INCLUSION OF \\ MINORITIES. LA CLASE MÁGICA, AN INTERVENTION \\ MODEL FOR SOCIAL CHANGE
}

\author{
Mayra Martínez Avidad \\ Universidad Camilo José Cela. Madrid, España \\ mmartinez@ucjc.edu \\ Olga A. Vásquez \\ Universidad de California, San Diego \\ Department of Communication Media \\ Center and Communication Building (MCC) CA, EEUU \\ ovasquez@ucsd.edu
}

\begin{abstract}
Resumen
Las minorías culturales y étnicas son con frecuencia los grupos más desfavorecidos en términos sociales y económicos debido a su falta de representación institucional. El objetivo de este artículo es proponer un modelo de intervención capaz de aumentar el poder de participación de una minoría a través de la comunicación horizontal con otras identidades mayoritarias. Los resultados del estudio de caso del programa socioeducativo La Clase Mágica, para el que se utiliza una metodología eminentemente cuantitativa a partir de cuestionario, demuestran que estos aspectos contribuyen a que los mexicanos de primera y segunda generación en el sur de California desarrollen capacidades clave para el empoderamiento y la inclusión social. Además, el programa impactó en un factor determinante para que una comunidad consiga ganar poder de acción y voz suficiente para influir en las instituciones sociales: los apoyos externos.
\end{abstract}

Palabras clave: Inclusión social, empoderamiento, minorías, comunicación para el cambio social, agentes sociales de cambio, contacto intergrupal, La Clase Mágica. 


\begin{abstract}
Cultural and ethnic minorities are typically among the socially and economically disadvantaged groups in most societies due to their lack of institutional representation. The aim of this paper is to propose an intervention model capable of increasing the participatory power of a minority group through sustained and strategically design interaction between them and representatives of the dominant groups in the society. The results of the case study of the socioeducational program La Clase Mágica, employing survey methods to collect data, shows that those elements contributes to develop key capabilities for the empowerment and social inclusion of the first and second generation Mexican origin immigrants in San Diego County. Furthermore, the results show that the program was a decisive factor in promoting community empowerment and voice in influencing social institutions in terms of external support.
\end{abstract}

Keywords: Social inclusion, empowerment, minorities, communication for social change, social agents of change, intergroup contact, La Clase Mágica

\title{
Extended abstract
}

Community underdevelopment is not limited to the poorest regions of the world. Disadvantage and social exclusion is also found in more developed and affluent societies. This is particularly true among cultural and ethnic minority populations who are often marginalized and underserved in advanced societies. The aim of the work presented here is to explore the opportunities of inclusion embodied in communication strategies purposively designed to engage excluded groups who have less access to resources and power. Specifically, this article presents an interventionist model of social change focused on promoting development and social inclusion through organized intergroup relations between representatives of majority and minority communities. We argue that this model promotes empowerment and social inclusion as a means of transforming power relations as well as social institutions and organizations. The project follows several lines of intervention aiming at empowering those who are poor and socially excluded. First, it pursues substantial economic and educational development in the community by providing access to cultural and economic capital necessary for social advancement. Moreover, this intervention aims at promoting the development of a critical consciousness and a new form of being in the world that reduces the differences in the distribution of resources that grow out of inequality in power and social status. These new attitudes are not only expected of the minority community but of members of the mainstream society as well. The development of a new consciousness is seen here as a key element for mobilizing underresourced communities -i.e. in the form of collective action- to not only come together, but to also serve as an inspiration that supports their social goals (tacit actions to support the minority group).

This model of social inclusion is based on the fundamentals of critical communication theory, social constructionism, as well as the psychological mechanisms for reducing discrimination and prejudice. The main contribution of critical communication theory proposed by the model is the conception that 
reality is intersubjectively constructed from agreements that people generate through dialogue. Communication determines action and this, in turn, changes social reality and culture (Bruner, 2000; Freire, 2003; Aubert et al., 2008; Castells, 2009). Moreover, the use of intergroup communication strategies as an incentive for change is not something new. Strategies to reduce discrimination experienced by minority groups in intergroup contact have been studied for over several decades. Such experiments have attempted to reproduce favorable conditions in which the dominant group re-assesses the stigmatized group and reduces its own privileged position -important antecedents for eliminating prejudice- (Pettigrew, 1998). From this perspective, nonhierarchical communication with members of disadvantaged groups can have a transformative social impact for the majority group as well. In fact, it is not uncommon to find individuals who support social causes that are not their own. Laclau (2005) calls this solidarity with the demands of others "chains of equivalence" and believes that it is the first condition to the formation of a collective agent.

The fundamental principles of this model are observable in La Clase Mágica, an educational program located in Latino communities in Southern California and Texas (U.S.A), where low-income Latino youth and undergraduate students hailing from all parts of the world collaborate within a nonhierarchical bilingual environment. The research presented here highlights the methodological principles of the program and the fact that its empirical findings have had a long term impact on the social inclusion and empowerment of participants. Our goal is to support the tentative hypothesis that the shift towards a more just and egalitarian society is sprouted in nonhierarchical communication between representatives of the majority society and of minorities groups at risk of exclusion.

The findings on program impact at La Clase Mágica reveal several indicators that are considered key to empowerment and the social inclusion of a minority group. Among these are income (economic capital), level of education (cultural capital), and critical (democratic participation and collective action) and social capacities (social support and the reduction of prejudice). A questionnaire was administered to four participant groups (1) Latino youth, (2) Latino adults, and two University of California participant groups: (3) researchers and doctoral students employed as staff by the university and (4) undergraduate students enrolled in a practicum course. In the case of the first three participant groups, the research incorporated all those who had participated in LCM continuously for at least two years, regardless of the time of entry. A sample of 41 undergraduated students who participated in LCM as part of the academic course for periods of approximately three months were also interviewed on the impact the program had had on their own personal lives.

The research findings indicate that this type of intergroup contact empowers the minority community to take action to improve its quality of life. The three pillars on which La Clase Mágica's socio-educational program is based and which have been instrumental in achieving its goal of social inclusion include: (1) educational and technology resources, (2) organized intergroup inter- 
actions around dialogue and collaboration, and (3) a non-hierarchical, intercultural and bilingual environment.

Two concrete research findings are particularly optimistic: All of the college aged youth, except one, were presently enrolled or had completed their college degree. Meanwhile, all Latino adults (except one) enjoyed a better paid job than before participating in La Clase Mágica. Relations with the university college provide Latino teens with the know-how necessary to pursue an academic life. The program also served to promote values of community participation and collective action. The data collected illustrated that the Latino community began to be aware of their influence to solve the problems plaguing their community. Some of them took part in local organizations or participated in actions of social or political activism.

Meanwhile, the university researchers who participated in La Clase Mágica came face to face with the great challenges facing minority communities in the United States. As a result of this experience, many decided to work to meet the needs of minority communities in the United States and/or social change through an educational perspective. Finally, the data also reflects a reduction in prejudice and an increase in positive attitudes toward immigrants by college students. For example, after the university students had had contact with the Mexican community, there was a significant decrease in the belief that immigrants took jobs away from Americans. Many also acknowledged that the contact with the community expanded their knowledge of Mexicans and influenced them to change their beliefs based on stereotypes of this community.

\section{INTRODUCCIÓN}

El campo de la comunicación para el desarrollo y el cambio social se ha ocupado desde sus inicios por describir procedimientos de actuación y metodologías capaces de promover el desarrollo de comunidades desfavorecidas en el mundo haciendo uso de estrategias comunicativas (ya sean medios de comunicación, formas de comunicación interpersonal o intercultural, o más recientemente, tecnologías de la información y las comunicaciones). Sin embargo, la amplia mayoría de las aportaciones teóricas e intervenciones en el campo han ido siempre dirigidas a aumentar los índices de desarrollo y aliviar de la pobreza las regiones pobres en el mundo. En muy pocas ocasiones los modelos descritos han sido optimizados para aplicarse en países ricos, donde todavía existen minorías cuyos índices de desarrollo (en términos de educación, ingresos, asistencia médica y hasta esperanza de vida) se equiparan o incluso colocan por debajo de los índices observados en países aún emergentes. El objetivo de este trabajo es ahondar en las oportunidades de inclusión social de aquellos grupos con menor acceso a recursos, debido a las desigualdades de poder y status en una sociedad dada haciendo uso de estrategias comunicativas. 
Aunque la acción colectiva y la movilización social son estrategias de abajo a arriba, consideramos que organismos e instituciones de índole pública y privada tienen un papel muy importante a la hora de dar respuesta a la problemática de la marginación social a través de programas específicos (Torres Valdés y Campillo Alhama, 2013). En concreto, este artículo plantea un modelo de intervención para el cambio social centrado en promover el desarrollo y la inclusión social de minorías a través de la comunicación intergrupal con miembros de la sociedad mayoritaria. Se parte de la premisa de que, para que tal cambio sea posible, la comunicación y el intercambio debe hacerse en un contexto, y bajo unas condiciones propicias, capaces de favorecer la formación de una coalición de cambio y una nueva conciencia crítica en ambos. Dicha toma de conciencia estimularía conductas dirigidas a corregir las diferencias en el reparto de recursos, incentivando por un lado la movilización de las comunidades desfavorecidas (procesos de acción colectiva) y, por otro, provocando una influencia social de apoyo a su causa (acciones de apoyo tácito al grupo minoritario). Para ello, la interacción y comunicación deben producirse en un contexto de igualdad de status y de colaboración hacia objetivos compartidos.

Este es precisamente el modelo que aplica La Clase Mágica, un programa socioeducativo para comunidades latinas en el sur de California y Texas, donde latinos de bajos recursos y americanos colaboran e interactúan en un entorno bicultural y sin jerarquías. La investigación que aquí se presenta ahonda en los principios metodológicos del programa y ofrece datos empíricos sobre el impacto a largo plazo del mismo en el empoderamiento e inclusión social de los participantes. Todo ello, con vistas a respaldar la hipótesis tentativa según la cual el cambio hacia una sociedad más justa e inclusiva pasa por fomentar una comunicación horizontal entre la sociedad mayoritaria y aquellas minorías en riesgos de exclusión.

\subsection{Fundamentos teóricos del cambio social}

La mayoría de los esfuerzos dirigidos a la inclusión social de minorías han estado encaminados a lograr que éstas aumenten su voz y control sobre las instituciones de su país. Las instituciones son elementos críticos para la reducción de la pobreza y la exclusión porque establecen las reglas del juego y con ello, el acceso a los recursos, las capacidades y oportunidades de las personas para avanzar socialmente hacia sus aspiraciones (North, 1990).

Incluso en las sociedades democráticas más avanzadas, la representación institucional -y, por siguiente, la posibilidad de establecer las reglas que definen el acceso a los activos y recursos de un país- varía de unos grupos a otros en base a las identidades sociales. Ello significa que la raza, cultura de origen, idioma, 
género, orientación sexual, ideología política, es decir, los elementos que constituyen una identidad social, determinan en última instancia el acceso a los recursos vitales, normalmente en detrimento de las identidades minoritarias (Bloemraad y Schönwälder, 2013).

Estos grupos con menos representación institucional tendrán menos oportunidades para conseguir cristalizar en la sociedad aquellas normas sociales, leyes, ideas (discursos en términos de Foucault) favorables a sus intereses. No obstante, según el propio Foucault (1991), esta lucha por el poder -esta lucha en última instancia por la hegemonía- es un proceso dinámico y sujeto a cambios. En este sentido, el orden social debe entenderse como una construcción histórica y contingente cuyo terreno es el discurso (Laclau, 1985). Dada la capacidad última de los individuos y grupos para aceptar, oponerse y combinar creativamente las normas y prácticas sociales, los miembros de una sociedad tienen algunas oportunidades para redefinir las reglas del juego. Como lo expresa más recientemente Castells (2009: 38): "Los conflictos nunca acaban, simplemente se detienen gracias acuerdos temporales y contratos inestables que son transformados en instituciones de dominación por los actores sociales que lograron una posición ventajosa en la lucha por el poder".

De esta manera, el empoderamiento puede considerarse como el proceso que permite a un actor social ganar poder de acción, así como capacidad para hacer escuchar su voz e influir en las instituciones (Oxby, 2009). Inclusión y empoderamiento son en este sentido dos fuerzas que se complementan y refuerzan mutuamente para inducir el cambio social (Bennett, 2002). Primero, una comunidad de excluidos se empodera cuando consigue ganar poder de acción y voz suficiente para conseguir un cambio institucional. Una vez que en la sociedad en la que vive dicha comunidad nacen instituciones que prescriben unas normas sociales favorables a sus intereses, se puede decir que se ha producido la inclusión social.

\subsection{Conciencia crítica, acción colectiva y empoderamiento}

Aunque existen múltiples propuestas que recogen indicadores cuantitativos y multidimensionales capaces de medir el nivel de exclusión y vulnerabilidad de un grupo social (Nabarro y Larrubia, 2006), existe sin embargo un déficit de concreción a la hora de señalar aspectos que contribuirían eficazmente al empoderamiento de un grupo social. Además de la importancia de la educación, así como de disponer de recursos económicos y materiales (ambos vistos como los máximos exponentes de la movilidad social), este artículo señala otro aspecto de crucial importancia para el empoderamiento de un grupo minoritario. Se considera que, conjuntamente con el capital económico y el capital cultural, es 
necesario disponer de capacidades críticas que le permitan a un grupo tomar conciencia de la relación de desigualdad en la que se encuentra y de las posibilidades de acción que tiene a su alcance para mejorar su situación.

Se parte de la premisa de que la adquisición de una conciencia crítica es un aspecto esencial para que los excluidos perciban la necesidad de emprender acciones colectivas que transformen su vida. Darse cuenta de que las propias condiciones de vida son el resultado de una injusticia ha sido, a lo largo de todos los tiempos, el motivo principal que ha llevado a la sublevación (en algunos casos a las armas) y el principio de muchas conquistas sociales (Bauman, 2009). En este sentido, la formulación de una demanda democrática por parte de un actor excluido del sistema surge de la conciencia de algún tipo de privación y, por siguiente, de la supuesta vulneración del principio de igualdad (Laclau, 2005).

Además de las estrategias de movilización y concienciación desde la base de la propia comunidad -actuaciones a menudo puestas en marcha a través de metodologías como la Movilización Social (Toro y Rodríguez, 2001) o la Comunicación para el Cambio Social (Gumucio-Dagron, 2010)- también la comunicación, entendida como un proceso intersubjetivo a través del cual se conforman las subjetividades individuales y colectivas, contribuye no solo a dotar de sentido a la realidad, sino también a cuestionarla y desafiarla. Las teorías comunicativas de Paolo Freire (2003) están en estrecha relación con esta visión. En este sentido, la comunicación horizontal entre distintos grupos sociales contribuye a poner de manifiesto el estado de privación y la contradicción existente entre las capacidades del grupo minoritario y aquellas a las que tienen derecho, lo que les incentivaría a movilizarse para alcanzar un reparto más equitativo ${ }^{1}$.

\subsection{Formación de agentes colectivos para el cambio: el apoyo social}

Además de las capacidades críticas señaladas, otro aspecto muy importante para el empoderamiento comunitario consiste en las relaciones sociales (capital social ${ }^{2}$ ) traducidas en apoyos internos como externos por parte de aque-

${ }^{1}$ Según la Teoría de la Privación Relativa, empleada precisamente para explicar movimientos subversivos "es más probable que estos actos se produzcan cuando los miembros de los grupos desfavorecidos perciban contradicción entre sus estado actual (capacidades) y aquel al que realmente tienen derecho (expectativas)" (Gil, 1999: 376).

${ }^{2}$ El capital social ya ha sido reconocido como importante activo de movilidad social por autores como Bourdieu (1983), Coleman (1988) y Woolock, M. (2001). Más recientemente autores como Fuchs (2011) han relacionado el capital social y las relaciones sociales con mayores niveles de participación democrática y la formación de acciones colectivas. 
llos actores sociales capaces de simpatizar con sus intereses. Dichos apoyos son de máxima importancia para que un grupo que se encuentra en una situación de desventaja comparativa consiga una mayor representatividad institucional. Aunque este trabajo no se inscribe completamente dentro de los límites de la Teoría de Movilización de Recursos ${ }^{3}$ (McCarthy y Zald 1977), sí otorga una crucial importancia al hecho de contar con los recursos y apoyos externos, especialmente aquellos institucionalizados, para que el descontento colectivo adquiera el peso social suficiente para lograr cambiar la realidad social y política.

En este sentido, el contacto comunicativo horizontal con grupos desfavorecidos puede suponer una influencia social transformadora también para las identidades mayoritarias. De hecho, no es en absoluto poco común encontrar actores que apoyan causas y demandas sociales que no le son propias. Laclau (2005) llamó a esta solidaridad con las demandas ajenas "articulación equivalencial" y supone, según el autor, la primera condición para la constitución de un agente colectivo.

Esta tendencia de los sujetos a implicarse por mejorar las condiciones de vida de grupos que se encuentran en una situación más desventajosa puede explicarse a partir de algunos supuestos psicológicos. Por ejemplo, según la Teoría de la Equidad, a las personas tampoco les gusta percibir que se benefician de un trato, posibilidades y oportunidades desproporcionadamente favorables frente al resto. En esos casos, experimentan reacciones de malestar o descontento, que tratarán de apaciguar emprendiendo acciones correctivas como el apoyo social (Hofmans, 2012). Desde el punto de vista emocional, el apoyo también se relaciona con un aumento de la autoestima que estimula una visión positiva de uno mismo (Stanfeld, 2006). El apoyo social se concretaría en acciones que benefician directamente a la causa que reivindica una comunidad desfavorecida. Entre ellas cabe señalar el apoyo organizativo, (recursos que fomenten la acción coordinada) apoyo político (acciones de activismo, protestas, asociacionismo en organizaciones que trabajan por la causa), apoyo institucional (ejercido por una persona pública en una institución formal de índole política, académica, empresarial o social).

Este trabajo señala precisamente este último aspecto de acción política coordinada y conjunta, así como la posibilidad de que las relaciones sociales se transformen en organizaciones de acción colectiva en torno a unas demandas concretas.

${ }^{3}$ Aunque la postura de este trabajo es algo menos determinista, (la sola movilización de recursos no condiciona el éxito de un movimiento social), se consideran que los movimientos sociales se desarrollan dentro de los límites de las oportunidades estructurales para el cambio y que dicho cambio dependerá de las capacidades para renegociar la posición en la estructura social. Algo para lo que resulta de vital importancia la cooperación y alianza con otros grupos de poder. 


\subsection{Contextos de mediación y comunicación intergrupales}

Muchas de las investigaciones realizadas en el campo de la psicología de los grupos han demostrado que las interacciones sociales regularizadas constituyen la base de muchos cambios e innovaciones en las sociedades (García Sáiz y Gil, 1999). Fruto de estas innovaciones, se constituyen nuevas formas de entender y concebir la realidad condicionando, en muchos casos, reivindicaciones o demandas sociales colectivas. La construcción de una demanda social involucra tanto a la subjetividad colectiva (entendida como aquellos procesos de creación de sentido sostenidos colectivamente), como a la articulación discursiva capaz de inscribir una situación como una demanda-deseo (Retamozo, 2009). En la construcción de cualquier sentido colectivo, no podemos sin embargo disociar la subjetividad de la intersubjetividad. La pedagogía crítica (Freire, 2003; McLaren y Kincheloe, 2008), pero también el interaccionismo simbólico (Blumer, 1982), la psicología cultural (Vigotsky, 1995; Cole, 1999; Bruner 2000), así como propuestas recientes en educación como el aprendizaje dialógico (Aubert et al., 2008; Racionero y Padrós, 2010), proporcionan excelentes marcos conceptuales para entender de qué manera la comunicación y la acción mediada conjunta afecta al desarrollo individual, social y cultural. Según estas perspectivas teóricas, la realidad es una construcción social que depende de los significados que establecen las personas a través de la comunicación. De esta manera, los significados no son una imposición social (que procede de afuera) sino más bien el fruto de los acuerdos entre los diferentes actores sociales, un acuerdo que se establece dialogando y comunicando, es decir, intersubjetivamente. Si la realidad es una construcción humana, una realidad hecha de significados que a su vez dependen de las interacciones humanas, la comunicación interpersonal e intergrupal es al mismo tiempo el mecanismo para alterar los significados que transformen la cultura, las conductas sociales y la sociedad misma.

Por otra parte, el empleo de estrategias de comunicación intergrupal como acicate para de cambio, tampoco es algo reciente. Desde hace ya varias décadas se han venido utilizando por ejemplo estrategias basadas en el contacto intergrupal para reducir la discriminación sufrida por las minorías (origen también de la marginación social). Los experimentos han tratado de reproducir las condiciones favorables en las que el grupo dominante consigue recategorizar al grupo estigmatizado y reducir el favoritismo endogrupal (importantes antecedentes del prejuicio). En esta línea Pettigrew (1998) propuso algunos requisitos necesarios para que el contacto produzca sus efectos: (a) aprender acerca del grupo minoritario (b) cambio de comportamiento (c) generación de 
lazos afectivos ${ }^{4}$, y (d) reevaluación del exogrupo. El mecanismo de reevaluación incluye una revisión de las creencias del propio individuo sobre el grupo de exclusión, parte de este proceso podría incluir un cuestionamiento de las prácticas sociales que benefician a un grupo sobre otro (McClelland y Linnander, 2006).

El modelo de intervención que proponemos parte, por tanto, de los supuestos teóricos previos, así como de las posibilidades para crear oportunidades para un contacto y un aprendizaje transformativo (Martínez Avidad, 2014).

\section{ESTUDIO DE CASO DE LA CLASE MÁGICA}

El modelo para el desarrollo y la inclusión social de minorías parte del esquema observado en el programa socioeducativo para comunidades latinas, La Clase Mágica (LCM) (Vásquez, 2003). Este programa nació en 1989 como una adaptación de la comunidad de aprendizaje La Quinta Dimensión (Cole, 2009), especialmente diseñada para responder a las necesidades específicas de los niños residentes en un barrio inmigrante de la ciudad de San Diego, California. Desde entonces, el proyecto ha recogido fondos de diversas fuentes locales y gubernamentales que le han permitido expandirse hasta llegar hoy día a seis centros repartidos por el condado de San Diego ${ }^{5}$. Más recientemente, se han replicado centros de LCM en San Antonio (Texas) Flores, (Vásquez y Clark, 2014), Bogotá, Colombia (Vásquez, 2015), así como en varias localizaciones en España: en la Universidad de Sevilla, Universidad Pablo Olavide y en la Universidad de Barcelona con poblaciones de gitanos (Macías Gómez-Estern y Vásquez, 2014,).

En La Clase Mágica los niños de origen mexicano desarrollan actividades educativas utilizando recursos tecnológicos en colaboración con estudiantes universitarios americanos de la Universidad de California, San Diego (UCSD), quienes se desplazan a los centros dos veces por semana como parte de los requisitos de un curso de practicum. Estos estudiantes aplican su conocimiento sobre el desarrollo educativo infantil trabajando de cerca con niños latinos.

${ }^{4}$ Sobre la importancia de las amistades entre grupos, Petigreew es el primero en sugerir que los sentimientos pueden ser más susceptibles al contacto que las propias creencias.

${ }^{5}$ En el momento del estudio, existían seis centros donde se desarrollaba La Clase Mágica en instituciones de la comunidad: dos colegios, dos centros comunitarios, una reserva indígena, además de la pequeña misión en Solana Beach. Todos estos centros estaban dirigidos por 10 coordinadores reclutados en la propia comunidad que trabajan en estrecha colaboración con el personal investigador de la UCSD, la cual proveía a su vez de 7090 universitarios matriculados en el practicum sobre desarrollo educativo durante el curso académico. 
Los estudiantes enseñan a los niños mexicanos a manejar los ordenadores y el software educativo y les ayudan con las tareas escolares. Al mismo tiempo, interactúan con los coordinadores del programa (los mexicanos adultos que dirigen las actividades diarias en los centros). Dado que estos estudiantes provienen en su amplia mayoría de familias de un nivel socioeconómico alto, este contacto con la comunidad de bajos recursos es una circunstancia excepcional en sus vidas. Junto con estos universitarios, la universidad aporta una red de investigadores y estudiantes de doctorado quienes desarrollan actividades de investigación en los centros. Se trata de personal contratado por la Universidad, normalmente estudiantes de doctorado para llevar a cabo labores de investigación y coordinación en los centros. Entre sus labores se encuentra realizar pruebas de evaluación, planificar el currículo académico y coordinar con los empleados mexicanos las tareas de los centros. En su trabajo aprenden a desarrollar entornos educativos óptimos y técnicas y metodologías de evaluación. Estos dos participantes provenientes de la universidad (universitarios e investigadores), que disfrutan de un estatus socioeconómico elevado, son representativos de la sociedad americana mayoritaria. Ambos interactúan, se comunican y relacionan con los niños latinos (segunda generación de inmigrantes), así como con los coordinadores de los centros, en su mayoría padres latinos de los niños participando en el programa. El perfil demográfico de estos padres corresponde a personas jóvenes (en su mayoría mujeres) de clase trabajadora con poca fluidez en inglés y sin apenas conocimiento en las tecnologías de la información (Vásquez, 2003). Aunque en principio estos padres se unieron al proyecto como voluntarios, pronto empezaron a percibir un salario por su trabajo en LCM. La principal labor de los padres latinos es crear nuevos materiales curriculares y gestionar las actividades diarias en los centros. El programa pretende contribuir a mejorar su situación económica gracias al propio trabajo que desempeñan en el programa y por medio de la formación y aprendizaje logrado durante su experiencia en LCM. Además, al pasar más tiempo con sus hijos en actividades académicas dirigidas, se persigue que los padres contribuyan positivamente en su rendimiento, a la vez que -del contacto con personal y estudiantes de la universidad-sean más conscientes de los beneficios de la educación universitaria y de la importancia del dominio de las tecnologías.

El proyecto propone dos líneas de intervención como fuentes principales de ampliación de oportunidades sociales y desarrollo comunitario: (1) recursos educativos y tecnológicos, (2) interacción, diálogo y colaboración intergrupal habilitado en un entorno bilingüe, intercultural y sin jerarquías. Estas líneas están dirigidas a generar competencias en los individuos participantes que 
contribuyan a ampliar sus oportunidades de mejorar su realidad social presente. El desarrollo y avance social de la comunidad latina descansa, tanto en esa comunicación y diálogo con miembros de la sociedad mayoritaria, como en el desarrollo de sus capacidades a través de la educación y el acceso a las tecnologías de la información y comunicaciones. El proyecto pone un énfasis sustancial en la educación como medio para el aumento de capacidades. Los niños aprenden a sumar, restar, dividir, leer en inglés y realizar análisis sintácticos haciendo uso de las tecnologías de la información. Esa preparación educativa aumenta sus oportunidades sociales futuras y amplía sus posibilidades de ascenso social. El entorno bicultural y bilingüe en el que se desarrolla el programa refuerza, además, la seguridad y autoconfianza de los latinos, otro elemento clave para su desarrollo futuro. La interacción de los propios latinos en un espacio común donde se preserva su idioma y los rasgos de su cultura de origen, refuerza los sentimientos de pertenencia, el sentido de comunidad y de identidad mexicana. La comunidad logra mejorar la conciencia de sí misma y de su situación, adquiriendo una identidad colectiva que aviva su compromiso por participar en acciones que benefician a la propia comunidad latina. Resulta esencial también el papel decisivo que se otorga a la propia comunidad para la coordinación y dirección de los centros. Son los propios mexicanos quienes se encargan de las tareas diarias de las escuelas de LCM, coordinan las actividades y deciden sobre muchos de los aspectos del programa. Por su parte, los participantes provenientes de la universidad estarían adquiriendo un mejor entendimiento del grupo minoritario y desarrollando, mediante la interacción regularizada, actitudes positivas hacia los inmigrantes Latinos. Este aprendizaje sobre el grupo de exclusión pretende contrarrestar la ignorancia acerca de la comunidad latina de inmigrantes y erradicar estereotipos. El programa también busca la creación de lazos afectivos y la familiaridad entre los participantes como un mecanismo para reducir el miedo y el recelo a menudo presente en las personas cuando tratan con inmigrantes y minorías.

\subsection{Material y métodos}

El estudio de caso trató de evaluar el impacto del programa La Clase Mágica en varios indicadores que se consideran clave para el empoderamiento, y por tanto, la inclusión social de una minoría. Entre ellos ingresos (capital económico), nivel de estudios (capital cultural) capacidades críticas (participación democrática y acción colectiva) y sociales (apoyo social y reducción del prejuicio). Para la investigación se entrevistó a cuatro grupos participantes: Dos provenientes de la minoría de bajos recursos: (1) adolescentes latinos y (2) adultos latinos; y otros dos miembros de la sociedad americana mayoritaria: (3) investigadores 
y estudiantes de doctorado empleados por la UCSD y (4) estudiantes universitarios de la UCSD. En el caso de los tres primeros participantes, la investigación se centró en todas aquellas personas que habían participado en LCM de modo continuado durante al menos dos años, sin importar el momento de su participación ${ }^{6}$. La muestra corresponde, por tanto, al universo total del colectivo estudiado: todas aquellas personas que participaron en LCM durante al menos dos años. Se administró un único cuestionario vía telefónica a 16 adolescentes de la comunidad, 10 adultos de la comunidad y 20 investigadores de la universidad. En el caso especial del cuarto participante, que participaba en el programa durante períodos de alrededor de tres meses, se seleccionó una muestra de 41 alumnos que participaron en LCM durante un curso académico.

Los cuestionarios dirigidos a la comunidad latina constaban de una primera parte donde se preguntaba sobre la influencia percibida del programa en sus logros académicos, sociales y económicos, etc. y una segunda donde se indagaba en los indicadores objetivos de empoderamiento e inclusión social. En esta segunda parte se preguntaba sobre niveles de educación alcanzados en el caso de los niños, nivel de ingresos en el caso de los adultos y participación en actividades de voluntariado en el caso de ambos. Las respuestas se organizaron en una escala Likert del 1 al 5 con la posibilidad de añadir comentarios que aportaron datos cualitativos sobre los que realizar más interpretaciones. El cuestionario dirigido a los investigadores de la universidad se centraba en las aportaciones del programa para formarles en su carrera de investigadores, así como en su impacto para ser más conscientes de la realidad social de estas comunidades, organizadas en preguntas y respuestas en una escala Likert del 1 al 5 y con posibilidad de añadir comentarios como base de un análisis cualitativo. Finalmente, con respecto a los estudiantes de la universidad, se administró el mismo cuestionario antes y después de su intervención en el programa, el cual contenía alrededor de 60 preguntas organizadas en una escala Likert del 1 al 5 sobre distintas percepciones en torno a los inmigrantes latinos, con el fin de observar variaciones en sus percepciones y actitudes tras su participación en LCM. Asimismo, al final de sus prácticas, se les pidió que comentaran brevemente si su experiencia en La Clase Mágica les había influido para tener un mejor conocimiento de la comunidad latina o si dicha influencia había servido para erradicar algunos estereotipos previos sobre los latinos.

\footnotetext{
${ }^{6}$ Así, puesto La Clase Mágica llevaba en funcionamiento alrededor de quince años, algunos de los niños que habían participado en el programa durante sus inicios serían adultos de entre 20 y 25 años en el momento de la investigación. Esto, lejos de ser un inconveniente, ayudaría a reflejar el impacto a largo plazo del programa en los logros académicos y sociales de los individuos participantes.
} 
Para el análisis de los resultados obtenidos a partir del cuestionario se hizo uso del paquete estadístico SPSS.

\subsection{Resultados}

\subsubsection{Adolescentes latinos}

Los resultados muestran que LCM tuvo una fuerte influencia en fomentar el interés de los niños latinos por cursar estudios universitarios. El 78\% afirmó que su participación les animó a ir a la Universidad entre "bastante" y "mucho". Entre aquellos que admitieron el impacto de La Clase Mágica en su éxito académico, la mayoría señaló la importancia de trabajar uno a uno con investigadores y estudiantes de las Universidad. Tal y como podemos observar de sus explicaciones en las entrevistas, el contacto y continua interacción con los estudiantes de la UCSD influyó positivamente a la hora de cultivar una visión favorable sobre la educación universitaria. En este contexto, los estudiantes actuaron como modelos para animar a los niños latinos a perseguir un futuro similar. Este sentimiento general puede ser observado en algunos de sus comentarios: J.G. de 25 años, quien participó en LCM cuando tenía 10 años de edad declaró que "los estudiantes universitarios me inspiraron mucho. Sabía que quería ser como ellos. Sobre todo Olga Vásquez, una latina de la UCSD ¡Caray! Ella me sirvió de modelo y me hizo pensar que un día podría lograr lo mismo". Los resultados revelaron que el casi el 66\% de los jóvenes latinos (antes niños participando en el programa) había completado un título universitario o estaba en ese momento matriculado en un programa universitario. Si tenemos en cuenta solo a los jóvenes en edad de ir a la universidad, el 90\% estaba, de hecho, cursando estudios universitarios o ya había obtenido sus diplomas, lo que supone un porcentaje muy elevado si se compara con la tasa de matriculación de los hispanos en estudios universitarios en Estados Unidos ${ }^{7}$. De las entrevistas también observamos a algunos de ellos interesados en realizar estudios de postgrado. De hecho, encontramos que más del 32\% planeaban firmemente realizar estudios de master o doctorado.

Casi el 70\% de todos los entrevistados disponía de un empleo estable en el momento de la entrevista. En cuanto a los ingresos percibidos, la mayoría (31\%) de los jóvenes se encontraban en la horquilla entre los 20.000 y los 30.000 dólares al año. Dos de los entrevistados ingresaban entre 30.000 y 40.000 dóla-

${ }^{7}$ De acuerdo con los datos de la oficina Censo de Estados Unidos (US Census Bureau), sólo el 7,6\% de los mexicanos de 25 años y más mayores poseían un diploma universitario, o estudios más avanzados en 2002. Es más, los datos muestran que el 32\% de los mexicanos mayores de 24 años en Estados Unidos no había llegado al noveno grado en el mismo año. 


\section{Gráficol. Impacto de LCM en el interés de los adolescentes latinos por cursar estudios universitarios}

¿Incrementó LCM tu interés por ir a la Universidad?

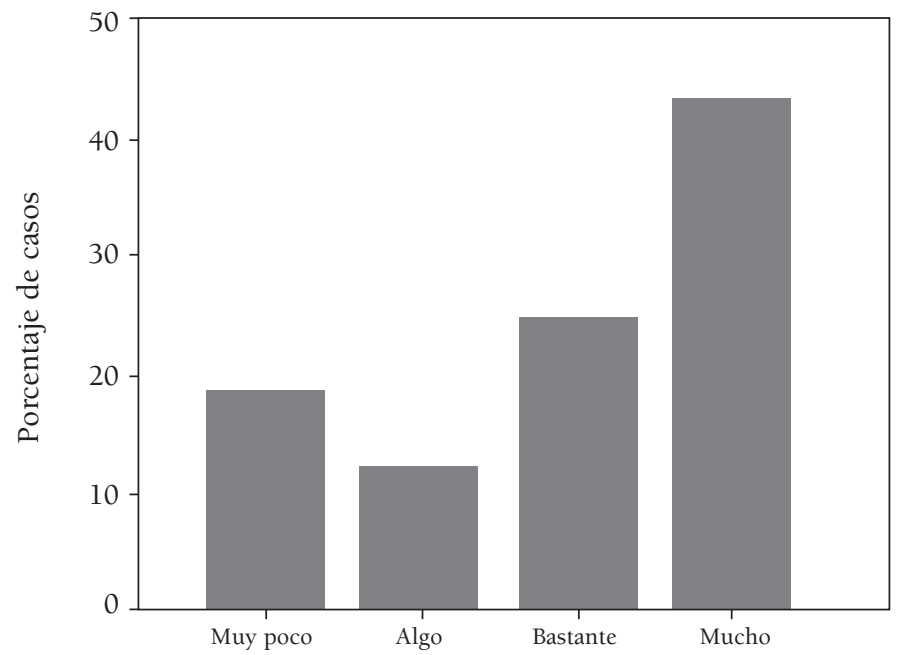

res al año. Unos ingresos apreciables, si tenemos en cuenta que se trata de jóvenes de entre 13 y 25 años (muchos de ellos son menores de edad) ${ }^{8}$. Además, los resultados revelan que su participación en el programa incrementó su interés por participar en el beneficio de la comunidad. Casi todos los jóvenes (95\%) que habían participado siendo niños en LCM opinaba que las personas debían involucrarse para favorecer a su comunidad. Una amplia mayoría (93\%) pensaba, de hecho, que podían contribuir a solucionar muchos de sus problemas locales si se involucraban de forma activa.

\subsubsection{Adultos latinos}

Por su parte, el 70\% de los coordinadores (adultos latinos) aseguraron que su situación económica había mejorado como resultado de su participación en LCM. La posibilidad de ser contratados como coordinadores de los centros suponía un primer paso a la hora de mejorar la situación profesional de estos participantes. El 90\% de las personas entrevistadas tenía un empleo fijo. Este alto porcentaje tiene implicaciones importantes, especialmente si tenemos en cuenta que el $90 \%$ del personal de la comunidad latina contratado por LCM

\footnotetext{
${ }^{8}$ Según el retrato estadístico de los hispanos en Estados Unidos realizado por el centro de investigación PewHispanic Reserach, la media de ingresos familiares de los hispanos en 2006 fue de 38.235 dólares al año.
} 


\section{Gráfico 2. Impacto de LCM en la mejora de condiciones laborales de los latinos adultos}

¿Su situación económica es mejor gracias a LCM?

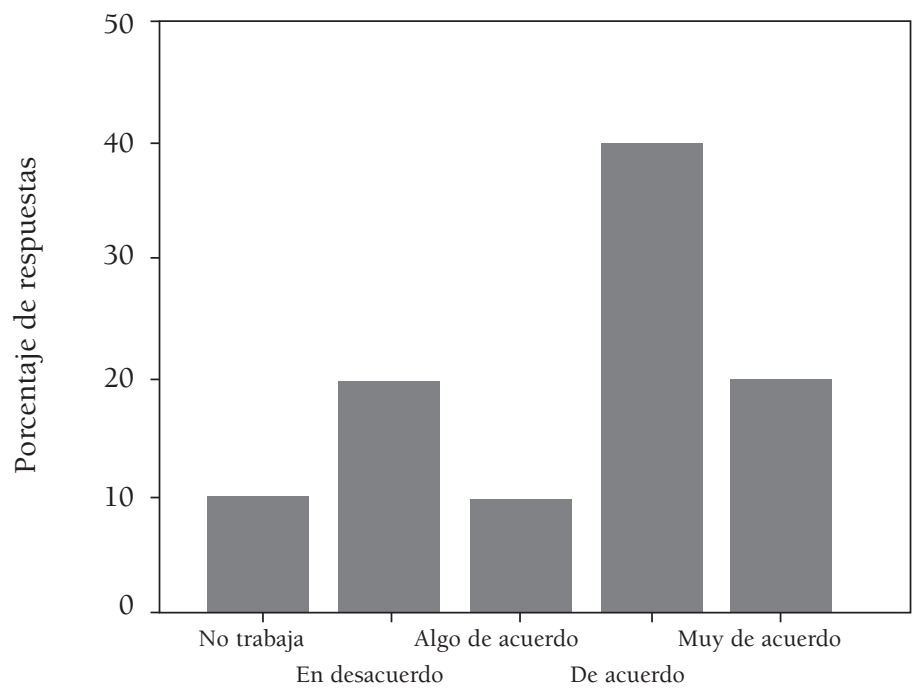

eran mujeres. V. G., de 48 años de edad en el momento del estudio, admitió que su experiencia como voluntaria en LCM y el apoyo de su hija, que también participaba en el programa como estudiante de la UCSD, le animó mucho a convertirse en maestra. "Antes trabajaba limpiando casas. Ahora soy increíblemente más feliz de ser capaz de trabajar con niños; Los adoro. Siempre fue mi sueño y La Clase Mágica me ha ayudado a cumplirlo".

Aunque sólo el 30\% y el 10\%, respectivamente, reconocía que participaba en algún tipo de organización o alguna forma de activismo social o político, más del $50 \%$ estaba involucrado en actividades de voluntariado dentro de su comunidad. Esta participación tenía lugar fundamentalmente dentro de la parroquia. Estas actividades reflejan la voluntad y determinación de participar en actividades que mejoraran el futuro de la comunidad y la calidad de vida de todos sus miembros. Resulta también indicativo que todos los participantes (100\%) que trabajan para conseguir una igualdad de oportunidades, fueran los mismos que admitían sentirse capaces de influir positivamente en los problemas locales de la comunidad. En este sentido, todos los entrevistados estuvieron "de acuerdo" (aunque en diferentes proporciones) acerca del papel positivo de LCM en proporcionar un mejor entendimiento de los recursos comunitarios. Todos ellos también percibían el programa como un estímulo muy positivo a la hora de animar a los latinos a participar en las actividades dentro de la comunidad. 


\subsubsection{Investigadores de la universidad}

El 75\% de la plantilla de estudiantes de la UCSD que participó activamente en el programa durante varios años continuó su trabajo investigador después de que trabajaron en LCM. De los 20 entrevistados, ocho poseía un título de doctorado y tres un título de master en el momento de la entrevista, mientras que dos estaban en esos momentos cursando un programa de doctorado y tres cursando un programa de master. Los resultados indican que el campo de investigación de la mayoría (65\%) de los investigadores empleados por la universidad estaba íntimamente relacionado con La Clase Mágica. Educación, idiomas, estudios culturales y étnicos eran los campos estrechamente relacionados con el programa. Más de la mitad del personal universitario entrevistado ganó interés por el ámbito de la educación y decidió poner en práctica su conocimiento trabajando para satisfacer las necesidades de las comunidades minoritarias en Estados Unidos y/o trabajar por el cambio social desde una perspectiva educativa. Los datos demuestran que 7 de los 20 entrevistados estaban en esos momentos trabajando para colegios o universidades en comunidades de bajos recursos. Participar en LCM supuso también una importante influencia para los investigadores y doctorandos de la universidad. Los resultados indican que el 65\% de los antiguos empleados universitarios estaban en

Gráfico 3. Acuerdo entre los investigadores de la UCSD sobre la importancia de trabajar ellos mismos por la igualdad de oportunidades

Es importante que yo trabaje por la igualdad de oportunidades

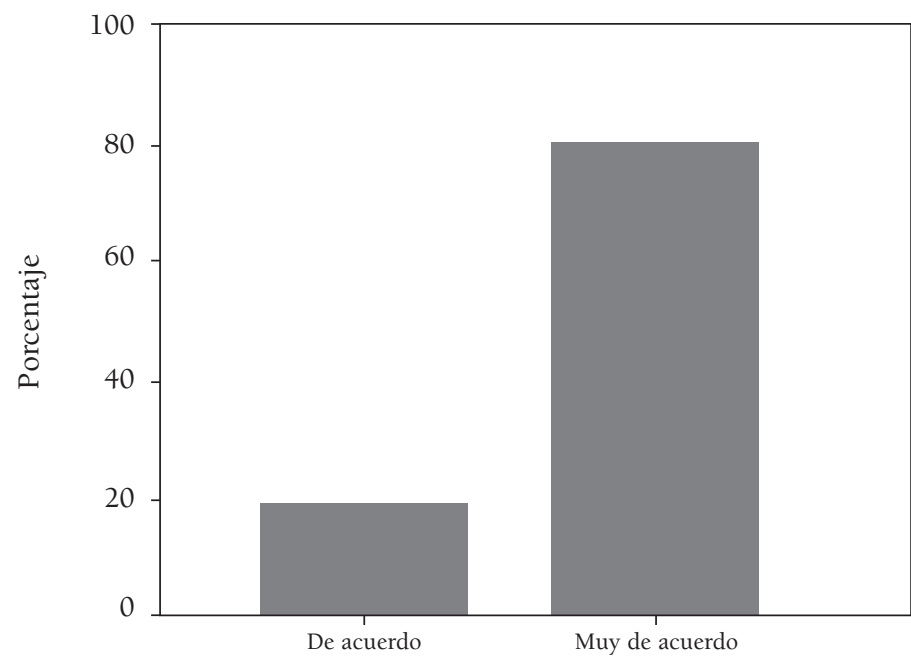


el momento del estudio participando como voluntarios en algún tipo de organización, involucrados en algún tipo de activismo político o en otras actividades de ciudadanía social orientadas a mejorar el bienestar de los latinos en Estados Unidos. Un ejemplo lo constituye un antiguo investigador que en el momento de la entrevista participaba activamente en el Centro Legal de La Raza, una agencia de servicios legales y de desarrollo comunitario cuya misión es dar apoyo a los latinos de bajos ingresos mediante una representación legal bilingüe. J. P. trabajaba para proporcionar apoyo legal sobre temas de empleo, derechos de estudiantes, inmigración, asistencia social, y protección al consumidor. "Muchos inmigrantes no hablan inglés o no conocen el sistema legal americano. Nosotros les proporcionamos una asistencia específica en cualquier cuestión legal y les ayudamos a conocer sus derechos", explicaba J.P.

\subsubsection{Estudiantes de la universidad}

De los resultados de la comparación entre el pre-cuestionario administrado antes de su participación en La Clase Mágica y el post-cuestionario administrado inmediatamente después de dicha participación (entre los cuales existe un intervalo de alrededor un mes y medio), puede observarse una variación significativa en 5 pares de ítems. Los resultados reflejan que disminuyeron significativamente los estudiantes universitarios de la UCSD que creían que los inmigrantes quitaban trabajo a los estadounidenses. Igualmente se observa que disminuyó significativamente el número de universitarios que estaba de acuerdo con que el Gobierno americano gastara más recursos para impedir que los inmigrantes ilegales entraran al país. El resto de variables que experimentan un cambio significativo tienen que ver con la extendida creencia entre los americanos de que la mayoría de los inmigrantes prefieren quedarse en Estados Unidos y convertirse en ciudadanos americanos y que por lo tanto no tienen interés de volver a sus países de origen.

Como complemento al estudio cuantitativo, se pidió a los alumnos que comentaran brevemente (de forma anónima si lo preferían) si pensaban que su experiencia en la comunidad latina les había hecho aprender algo sobre la comunidad o contribuido a erradicar algunos de los estereotipos que albergaban sobre los latinos. Casi el 70\% de los entrevistados admite en sus comentarios que el contacto con la comunidad amplió su conocimiento sobre la comunidad latina e influyó a la hora de erradicar ideas previas basadas en estereotipos. Algunos de los comentarios subrayan un marcado cambio de actitudes. Por ejemplo, algunos estudiantes reconocieron que antes de su experiencia en La Clase Mágica solía criminalizar inconscientemente a los latinos y albergaba miedos y creencias de que la mayoría pertenecía a bandas. K.R relata en sus comen- 
tarios que "Durante mi primer acercamiento al centro estaba llena de nerviosismo. Me hacía sentir incómoda el entrar en un aula de la comunidad latina. De mis experiencias en el instituto sabía que los latinos se juntaban entre sí y me daban mala impresión pues había rumores por ahí de que muchos pertenecían a bandas. Sin embargo, entré en la clase por primera vez un miércoles por la mañana y todos los miedos se disiparon. Estos niños de 3 a 5 años no tenían una vida predestinada y eran sólo niños con ganas de aprender".

Otros estudiantes reconocían que el contacto les había ayudado a modificar estereotipos comunes sobre la comunidad latina, como aquellos que les describen como personas perezosas y poco interesadas por aprender inglés, educarse o integrase en la sociedad americana. En algunas ocasiones, los estudiantes de la Universidad de California reconocían que su contacto directo con los mexicanos les había hecho aprender sobre la comunidad, corregir algunas ideas equivocadas y ser conscientes de algunos atributos positivos hasta el momento desconocidos sobre la comunidad latina. De los comentarios es posible también apreciar que las relaciones con niños y adultos mexicanos en La Clase Mágica habían desencadenado reacciones de empatía y amistad que habían transformado muchas de las actitudes (y posiblemente a partir de ahora conductas) hacia los inmigrantes.

\section{DISCUSIÓN Y CONCLUSIONES}

Las bases teóricas para el cambio social se inscriben en las relaciones de poder construidas sobre la base de la identidad social y el estatus económico y que a su vez están mediadas por las instituciones sociales. Éstas prescriben las normas y leyes que determinan el acceso a los recursos vitales para cada una de las identidades sociales en una sociedad, siendo las minorías las más perjudicadas en dicho reparto por su, a su vez, limitada influencia en dichas instituciones. El modelo de intervención propuesto se dirige a activar el proceso de empoderamiento que permita a las minorías aumentar su influencia y su voz. La inclusión social sería fruto de esta influencia.

Inspirado en los fundamentos de la psicología cultural, la pedagogía crítica y el aprendizaje dialógico, el modelo coloca en el centro mismo de la intervención al proceso comunicativo por ser el origen de las subjetividades y de los significados que dan a la estructura social. El modelo propone, así, un diálogo horizontal y sin jerarquías entre la comunidad minoritaria y la mayoritaria en un contexto óptimo en el que la reconstrucción de sentido se oriente a la solidaridad y cohesión intergrupales.

Los principios distintivos del modelo parten de suponer que los cambios en la estructura social no sólo dependen de capacitar al grupo minoritario, 
sino de inducir una serie de cambios en la comunidad mayoritaria comenzando por la simple reducción del prejuicio, pero aspirando también a producir actitudes de apoyo tácito al grupo excluido. El papel de la comunicación en este proceso resulta crucial, ya que es la interacción humana, el diálogo y la comunicación entre los diferentes grupos sociales el elemento clave capaz de elevar el nivel de concienciación sobre problema de la exclusión social.

En este sentido, La Clase Mágica proporciona un contexto de colaboración e interacción óptimo entre la cultura minoritaria y mayoritaria el cual fomenta la formación de relaciones sociales, así como actitudes de acción colectiva. Los espacios de interacción de LCM reproducen la condiciones óptimas para la reducción del prejuicio al mismo tiempo que fomentan la creación de relaciones intergrupales basadas en la colaboración y provocando vínculos de amistad y confianza que desencadenan respuestas de apoyo social. Consiste en el diseño de espacios educativos innovadores dentro de la comunidad (en ocasiones dentro de escuelas locales) mediante la participación de los dos grandes agentes sociales que conforman la comunidad de aprendizaje: la propia comunidad (niños y padres latinos) y la comunidad universitaria de la Universidad de California, San Diego (universitarios e investigadores).

Los dos ejes en los que se centra el programa socioeducativo La Clase Mágica: (1) recursos educativos y tecnológicos, (2) interacción, diálogo y colaboración intergrupal habilitado en un entorno bilingüe, intercultural y sin jerarquías, contribuyeron a elevar muchos de los índices propuestos en este artículo para alcanzar inclusión social. Aunque el estudio no ahonda en si la comunidad latina consiguió finalmente alcanzar una mayor influencia en las instituciones sociales clave en California, la investigación demuestra que la mayoría de los participantes disponen de un poder de participación y de una capacidad de hacer escuchar su voz superior a la mayoría de los latinos en Estados Unidos. Los más elevados niveles de estudios de los niños latinos; la relativamente mejor situación económica de los adultos; su mayor compromiso por mejorar las condiciones de vida de la comunidad; y el apoyo tácito de la mayoría de los americanos pertenecientes a la sociedad dominante; hacen pensar, al menos, que la comunidad latina participante en LCM se encuentra en la senda para conseguir influir, promover y negociar un mejor acceso a los recursos que mejoren su vida.

\section{REFERENCIAS}

Aubert, A., Flecha, A., García, C., Flecha, R., Racionero, S. (2008). Aprendizaje dialógico en la sociedad de la información. Barcelona: Hipatia Editorial.

Bauman, Z. (2009). El arte de la vida. De la vida como obra de arte. Barcelona: Paidós. Bennett, L. (2002). "Using empowerment and social inclusion for pro-poor growth: a theory of social change". World Bank, Social Development Strategy paper. 
Bloemraad, I., Schönwälder, K. (2013). Immigrant and ethnic minority representation in Europe: Conceptual challenges and theoretical approaches. West European Politics, 36, 564-579.

Blumer, H. (1982). El Interaccionismo simbólico, perspectiva y método. Barcelona: Hora D.L.

Bourdieu, P. (1986). The forms of capital. En J. C. Richardson (Ed.). Handbook of theory and research for the Sociology of Education (pp. 241-258). New York: Greenwood Press.

Bruner, J. (2000). La educación, puerta de la cultura. Madrid: Visor.

Castells, M. (2009). Comunicación y poder. Madrid: Alianza editorial.

Cole, M. (1999). Psicología Cultural. Madrid: Morata.

Cole, M. (2009). Designing, implementing, sustaining and evaluating ideocultures for learning and development: The case study of the Fifth Dimension. En B. Sevda \& A. Aksukoc (Eds.). Perspectives on Human Development, family and culture (pp. 331-349). New York, NY: Cambridge University Press.

Coleman, J. C. (1988). Social capital in the creation of human capital. American Journal of Sociology, 94, 95-120.

Flores, B. B., Vásquez, O. A., Clark, E. R. (2014). Generating transworld pedagogy: Reimagining La Clase Mágica. New York: Lexington Publishers, Rowman Littlefield publishing group.

Foucault, M. (1991). Microfísica del poder. Madrid: La Piqueta. (Obra original publicada en 1979).

Freire, P. (2003). Pedagogía del oprimido. Madrid: Siglo XXI. (Obra original publicada en 1970).

Fuchs, C. (2011). Foundations of Critical Media and Information Studies. New York: Routledge.

García Sáiz, M., Gil, F. (1999). Procesos de influencia social. En F. Gil y C. M. Alcover (Eds.). Introducción a la psicología de los grupos (pp. 251-279). Madrid: Pirámide.

Gil, F. (1999). Relaciones intergrupales. En F. Gil y C. M. Alcover (Eds.). Introducción a la psicología de los grupos (pp. 357- 386). Madrid, Pirámide.

Gumucio-Dagron, A. (2011). Comunicación para el cambio social: clave del desarrollo participativo. Signo y Pensamiento, 58, 26-39.

Hofmans, J. (2012). Individual differences in equity models. Psycologica, 33, 473-482.

Laclau, E. y Mouffe, C. (1985). Hegemony and socialist strategy. Towards a radical democratic politics. London: Verso.

Laclau, E. (2005). La razón populista. Buenos Aires: Fondo de Cultura Económica.

Martínez Avidad, M. (2014). La Clase Mágica: Creating opportunities for transformative learning. En B. B. Flores, O. A. Vásquez y E. R. Clark (Eds.). Generating transworld pedagogy: Reimagining La Clase Mágica (pp. 33-47). New York: Lexington Publishers, Rowman Littlefield publishing group.

Macías Gómez-Estern, B. y Vásquez, O. (2014). La Clase Mágica goes international: Adapting to new sociocultural contexts. En B. B. Flores, O. A. Vásquez y E. R. Clark (Eds.). Generating transworld pedagogy: Reimagining La Clase Mágica (pp. 193209). New York: Lexington Publishers, Rowman Littlefield publishing group. 
Navarro, S. y Larrubia, R. (2006). Indicadores para medir situaciones de vulnerabilidad social: propuesta realizada en el marco de un proyecto europeo. Baética, 28, 485-506.

McCarthy, J., Zald, M. (1977). Resource mobilization and social movements: A partial theory. American journal of sociology, 86, 1212-1241.

McLaren, P., Kincheloe, J. (2008). Pedagogía crítica: De qué hablamos, dónde estamos. Barcelona: Graó.

McLelland K., Linnander E. (2006). The role of contact and information in racial attitude change among white college students. Sociological Inquiry, 76, 81-115.

North, D. (1990). Institutions, institutional change and economic performance, Cambridge: Cambridge University Press.

Oxoby, R. (2009). Understanding social inclusion, social cohesion, and social capital. International Journal of Social Economics, 36, 1133-1152.

Pettigrew, T. (1998). Intergroup contact theory. Annual review of psychology, 49, 65- 85.

Racionero, S., Padrós, M. (2010). The Dialogic Turn in Educational Psychology. Journal of Psychodidactics, 15, 143-162.

Retamozo, M. (2009). Las demandas sociales y el estudio de los movimientos sociales. Cinta Moebio, 35, 110-127.

Stansfeld, S. (2006). Social support and social cohesion. En Marmot M. \& Wilkingson R. G. (Eds). Social determinants of health (pp. 155-178). Oxford: Oxford, University Press.

Salazar, R., Vásquez, O. A., Mehan, H. (1996). Engineering success through institutional support. En A. Hurtado, R. Figueroa y E. García (Eds.). Strategic interventions in education: Expanding the Latina/Latino pipeline (pp. 100-137). Santa Cruz, CA: University of Santa Cruz.

Toro, B., Rodríguez, M. (2001). La comunicación y la movilización social en la construcción de bienes públicos. Bogotá: Banco interamericano de desarrollo.

Torres, R. M., Campillo, C. (2013). Desarrollo local y relaciones públicas para grupos desfavorecidos en la Comunidad de Madrid. Prisma Social, 10, 394-432.

Vásquez, O. (2003). La Clase Mágica: Imagining Optimal Possibilities in a bilingual learning community. New Jersey: Laurence Erlbaum Publishers.

Vygotsky, L. (1995). Pensamiento y Lenguaje. Barcelona: Paidós.

Woolock, M. (2001). The place of social capital in understanding social and economic outcomes. Isuma: Canadian Journal of Policy Research, 2, 1-17.

MAYRA MARTÍNEZ AVIDAD es doctora en Sociología de la Comunicación y Profesora en la Universidad Camilo José Cela de Madrid. Fue durante dos años visiting student (Becaria de Postgrado Fundación Caja Madrid) en la Universidad de California, San Diego. Especialista en el ámbito de la comunicación social, investiga los fundamentos teórico-prácticos de la comunicación para el cambio social. Particularmente estudia la influencia de la comunicación alter- 
nativa, digital, participativa y comunitaria en la constitución de la realidad social y política.

OLGA A. VÁSQUEZ, Profesora Asociada en el Departamento de Comunicación de la Universidad de California, San Diego, es una académica especializada en el ámbito de la investigación comunitaria. Particularmente trabaja en contextos extraescolares que se enfocan en el diseño de ambientes de aprendizaje apoyados en pedagogías asistidas por tecnología. La profesora Vásquez estudia cómo esos espacios de aprendizaje estratégicamente diseñados pueden alcanzar los objetivos deseados de optimizar el aprendizaje y desarrollo de los jóvenes de lenguas minoritarias.

Recibido: 02/11/15

Aceptado: 06/06/16 